\title{
Foot Operated Vehicle for Armless People
}

\author{
${ }^{1}$ Sachin R. Jadhav, ${ }^{2}$ Ajinkya Patil, ${ }^{3}$ Mayank Gangan, ${ }^{4}$ Shubham Patil, ${ }^{5}$ Siddhant Godam \\ ${ }^{1}$ Assistant Professor, \\ 2,3,4.5 UG Students \\ Department of Mechanical Engineering, \\ TSSM's Padmabhushan Vasantdada Patil Institute of Technology \\ Bavdhan, Pune-411021, Pune, India
}

\begin{abstract}
Transportation has become an integral part of people's day to day life. At certain times, in large countries like India, people are forced to travel long distance from their work place to their place of residence. People with upper limb amputation and hands have difficulties in travelling and cannot travel these long distances. They use devices such as wheel chair, crutches and artificial limbs for mobility. These however cannot be used for long distance outdoor transportation Therefore, the aim of this project is to design and fabricate Foot operated system for armless people. The contents include a description of the design and selection process including all important information about the vehicle. Due to rapid industrialization and development of the economy the expectation of the customer and their ability and willingness to pay for the product has changed drastically.
\end{abstract}

Keywords: Foot controller steering, Handicapped, Steering system

\section{INTRODUCTION}

Now a day's transportation has become great difficulty to and individual to reach the destination on time. Everyone has their own vehicle and people with all body parts are fortunate .It is unfortunate for partially disable people with hands. Disability is the repercussion of an impairment which can be mental, physical, emotional, vision, sensory. Disabilities can occur in upper extremities and in lower extremities. These people become more defendants and lose their confidence. Due to this effect, they stand a great disadvantage in using public as well as private transportation facilities. Foot Operated Steering vehicle was something new to come up with and we had an interest to make something innovative.

The main objective of the project is to design a foot operated system for handicapped people. To suit the requirements of disabled drivers with these special circumstances, this high technology solution allows for the vehicle to be steered using the left leg through a rotating footplate. There is no use of the arms in using primary driving controls. A national level survey conducted in India by the Central Government of India once in ten years revealed that, around 27 million people which are about $2.21 \%$ of the Indians are differently able. Among them, around 14.98 million were men while 11.84 million were women. Thus, the percentage of disabled people in rural area was higher than those in urbanareas.

\subsection{PARAMETER OVERVIEW}

Weight distribution: The weight distribution is the ratio of the horizontal weight displaced between the forward and aft wheels. It dictates how a trike handles and how stable it will be. The more weight on the forward wheels, the better the cornering and less oversteer. However, too much weight on the front wheel causes the rear wheel to be too light. In a vehicle which relies on gravity in some way, weight distribution directly affects a variety of vehicle characteristics, including handling, acceleration, traction, and component life. For this reason weight distribution varies with the vehicle's intended usage. For example, a drag car maximizes traction at the rear axle while countering the reactionary pitch-up torque. It generates this counter-torque by placing a small amountof counterweight at a great distance forward of the rear axle. In the airline industry, load balancing is used to evenly distribute the weight of passengers, cargo, and fuel throughout an aircraft, so as to keep the aircraft's center of gravity close to its center of pressure to avoid losing pitch control. In military transport aircraft, it is common to have a loadmaster as a part of the crew; their responsibilities include calculating accurate load information for center of gravity calculations, and ensuring cargo is properly secured to prevent itsshifting.

In large aircraft and ships, multiple fuel tanks and pumps are often used, so that as fuel is consumed, the remaining fuel can be positioned to keep the vehicle balanced, and to reduce stability problems associated with the free surface effect. In the trucking industry, individual axle weight limits require balancing the cargo when the gross vehicle weight nears the legal limit.

Center of gravity: Center of gravity is as crucial as weight distribution for optimum trike handling characteristics. However, unlike weight distribution, the center of gravity has no handling or stability compromises when its ratio is lowered. If all the weight is placed well below the axle, the trike is going to have excellent handling regardless of weight distribution. A uniform gravitational field the center of gravity is identical to the center ofmass, a term preferred by physicists. The two do not always coincide, however. For example, the Moon's center of 
mass is very close to its geometric center (it is not exact because the Moon is not a perfect uniform sphere), but its center of gravity is slightly displaced toward Earth because of the stronger gravitational force on the Moon's near side. The location of a body's center of gravity may coincide with the geometric center of the body, especially in a symmetrically shaped object composed of homogeneousmaterial.

An asymmetrical object composed of a variety of materials with different masses, however, is likely to have a center of gravity located at some distance from its geometric center. In some cases, such as hollow bodies or irregularly shaped objects, the center of gravity (or center of mass) may occur in space at a point external to the physical material-e.g., in the center of a tennis ball or between the legs of a chair. Published tables and handbooks list the centers of gravity for most common geometric shapes. For a triangular metal plate such as that depicted in the figure, the calculation would involve a summation of the moments of the weights of all the particles that make up the metal plate about point A. By equating this sum to the plate's weight $\mathrm{W}$, multiplied by the unknown distance from the center of gravity $\mathrm{G}$ to $\mathrm{AC}$, the position of $\mathrm{G}$ relative to $\mathrm{AC}$ can be determined. The summation of the moments can be obtained easily and precisely by means of integral calculus.

Wheel Track: The wheel track is the width between the two front wheels. The wider the wheel track the less susceptible the vehicle is to capsizing during cornering. However, if too wide, the vehicle becomes impractical on most bike lanes. A 32-inch wheel track offers excellent handling and is practical for all bike roads too. The fitting of aftermarket wheels to your vehicle may result in the vehicle's wheel track being altered. If the wheels you fit have a different offset, compared with the original wheels, then the wheel track will change. Wheel track is the distance measured across an axle from the centre line of one tyre tread to the centre line of the opposite tyre tread.

\section{PROBLEM STATEMENT}

For the disable-bodies, the task of selecting the right bike or any transport vehicle can be a difficult prospect. So you may think that choosing a bike for a disable person would be much more difficult. However, for those with special driving needs. This product is designed to assist people with disabilities to independently drive a vehicle. This project supports the rider's disability by helping them balance the vehicle, be effortless to operate and also looking and functioning like a main stream bicycle. This helps the rider gain the independence and confidence to ride a vehicle without assistance. Although this vehicle has been designed specifically for people with disabilities. They are not allowed to move all around by themselves because of the reason of the inability. Many vehicles have been made on the disabilities for the people with no legs, but there is no vehicle particularly made for the armlesspeople.

\section{DESIGN OF COMPONENTS}

\subsection{Design of Frame}

In the first step we have designed the frame of the project Frame is a skeleton upon which parts like engine are mounted. So it is important that the frame should not buckle on uneven road surface.

We have designed -ROLL CAGElframe. Material Used For Frame: Mild Steel.

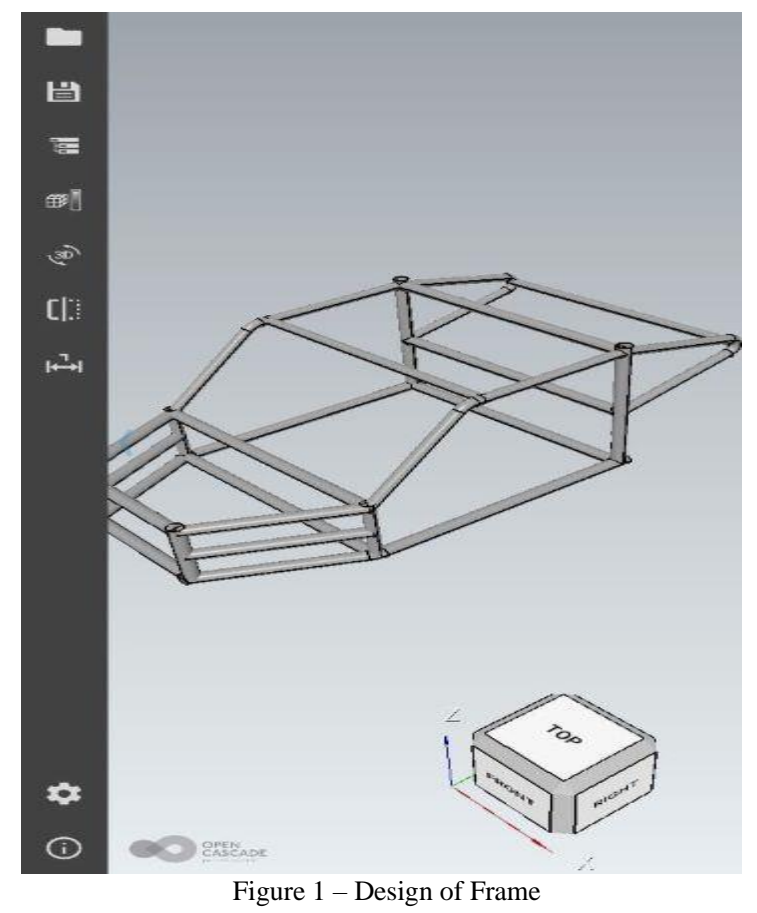

\subsection{Suspension System}

Suspension allows relative motion in cars. The design of spring is to verify by using customize package of ANSYS, HYPERMESH to perform finite element analysis. The static stress analysis is Perform and the results are comparing with theoretical calculations. Double Wishbone Suspension is an independent suspension design using two (occasionally parallel) wishbone-shaped arms to locate the wheel. Each wishbone or arm has two mounting points to the chassis and one joint at the knuckle. The shockabsorber and coil spring mount to the wishbones to control vertical movement. Double wishbone designs allow the engineer to carefully control the motion of the wheel throughout suspension travel, controlling such parameters as camber angle, casterangle, toe pattern, roll center height, scrub radius, scuff and more. An unequal length double wishbone suspension works by having an upper control arm that's shorter than the lower one. With this configuration, when the wheel travels upward, the top of the wheel tips inward, gaining negativecamber.

The double wishbone suspension provides the engineer more design choices than some other types do. 
It is fairly easy to work out the effect of moving each joint, so the kinematics of the suspension can be tuned easily and wheel motion can be optimized. It is also easy to work out the loads that different parts will be subjected to which allows more optimized lightweight parts to be designed. They also provide increasing negative camber gain all the way to full jounce travel, unlike the MacPherson strut, which providesnegativecambergainonlyatthebeginningofjounc etravelandthenreverses into positive camber gain at high jounce amounts. These wishbones are connected to the vehicle chassis from the wheels, and they use ball joint for the support and for the movement of the wheel for steering. The upper arm and the lower arm are usually of different size known as the short and long arm (SLA), this helps the wheel to gain proper camber angle during the wheel travel. The suspension spring is connected to the upper or lower arm and to the chassis of the vehicle. The spring and the damper system is used to dissipate maximum amount of energy in the spring and damper system from getting transferred to the chassis. And they increase the ride quality of the vehicle.

\subsection{Sterring System}

The pinion gear is attached to the steering shaft. When you turn the steering rod, the gear spins, moving the rack. The tie rod at each end of the rack connects to the steering arm on thespindle.

\subsection{Combine Brake and Acclerationpadel}

A COMBINED PEDAL MECHANISM is designed to function as both BRAKE and ACCELERATOR. This new mechanism enables the driver to control acceleration and braking using one foot, which will lead to reduction in stopping distance, mis-judgement and ultimately decrease in number of road accidents that may happen each day. The design of new mechanism aims, to provide a pedal that applies the brakes instantaneously and also permits the operation of accelerator with the same foot.

\subsection{Power System}

Lead Acid Battery is used in this electric car.

\section{6 toggle switch}

A toggle switch is a class of electrical switches that are manually actuated by a mechanical lever, handle, or rocking mechanism. Toggle switches are available in many different styles and sizes, and are used in numerous applications. Many are designed to provide the simultaneous actuation of multiple sets of electrical contacts, or the control of large amounts of electric current or mains voltages.

\section{CONCLUSION}

Hence we conclude that the project is done with the pure intention of providing a vehicle for the armless people and provide them with a proper vehicle with no dependence on any other human. The parameters that are described are all properly studied and then implemented in the experimental work of the manufacturing of thetricar

.all the designing is done for the proper analysis of the model. The calculations are primarily done by taking into consideration many of the parameters and then we have implemented in the manufacturing and the testing of the tricar.

\section{REFERENCES}

[1] Manish M. Patil (2017), Design \& Development for Three Wheeler Chassis, International Conference on Emanations in Modern Technology \& Engineering.

[2] Mr. Praveen D. Dethan, Syam M. Nair, NanduVijayakumar, Sarath Kumar (2017), Foot Controlled Steering System, Internation Journal of Machanical\& Industrial Technology.

[3] M.A.Saeedi, Stability of Three Wheeler Vehicle with \& without control system.

[4] Kumbhar Vikas S, NaganeSwapnil S, PatilSuraj Y, PawarRohit B (2015), Handfree Vehicle for Disabled, IJSRD.

[5] Mandar Sonam, SumitSonekar, SaurabhUpadhye (2015), Foot Operated Steering For Disabled People, IJMET.

[6] MandarPatil, PrashantPawar, SwapnilPatil, SwapnilHonroa (2016), Foot Operate Steering Machanism, IERJ.

[7] KatariRamaiah, An Efficient Car Driving Controller System Design For Physically Challenged People Using Arm Processor, International Journal of Engineering \& Science Research.

[8] Master of Science in Mechanical Engineering By Azadeh, Reference Book of Automobile Engineering (Volume $1 \& 2$ ) by Dr. Kripal Singh. 
